\title{
Outcomes of an inpatient refeeding protocol in youth with Anorexia Nervosa and atypical Anorexia Nervosa at Children's Hospitals and Clinics of Minnesota
}

Kathryn Smith ${ }^{1 *}$ (D) Julie Lesser ${ }^{2}$, Beth Brandenburg ${ }^{2}$, Andrew Lesser $^{3}$, Jessica Cici ${ }^{4}$, Robert Juenneman ${ }^{2}$, Amy Beadle ${ }^{2}$, Sarah Eckhardt ${ }^{2}$, Elin Lantz ${ }^{5}$, James Lock ${ }^{6}$ and Daniel Le Grange ${ }^{7}$

\begin{abstract}
Background: Historically, inpatient protocols have adopted relatively conservative approaches to refeeding in Anorexia Nervosa (AN) in order to reduce the risk of refeeding syndrome, a potentially fatal constellation of symptoms. However, increasing evidence suggests that patients with AN can tolerate higher caloric prescriptions during treatment, which may result in prevention of initial weight loss, shorter hospital stays, and less exposure to the effects of severe malnutrition. Therefore the present study sought to examine the effectiveness of a more accelerated refeeding protocol in an inpatient AN and atypical AN sample.

Methods: Participants were youth (ages 10-22) with AN $(n=113)$ and atypical AN $(n=16)$ who were hospitalized for medical stabilization. A retrospective chart review was conducted to assess changes in calories, weight status (percentage of median BMl, \%mBMI), and indicators of refeeding syndrome, specifically hypophosphatemia, during hospitalization. Weight was assessed again approximately 4 weeks after discharge.

Results: No cases of refeeding syndrome were observed, though $47.3 \%$ of participants evidenced hypophosphatemia during treatment. Phosphorous levels were monitored in all participants, and $77.5 \%$ were prescribed supplemental phosphorous at the time of discharge. Higher rates of caloric changes were predictive of greater changes in \%mBMI during hospitalization. Rates of caloric and weight change were not related to an increased likelihood of re-admission.

Conclusions: Results suggest that a more accelerated approach to inpatient refeeding in youth with AN and atypical AN can be safely implemented and is not associated with refeeding syndrome, provided there is close monitoring and correction of electrolytes. These findings suggest that this approach has the potential to decrease length of stay and burden associated with inpatient hospitalization, while supporting continued progress after hospitalization.
\end{abstract}

Keywords: Anorexia Nervosa, Refeeding, Hypophosphatemia, Children, Adolescents

\section{Plain English Summary}

Anorexia Nervosa (AN) has been shown to be the deadliest mental disorder due to the serious medical and psychiatric problems that are associated with this illness. Many hospital treatment programs start patients with AN on low calorie diets and increase their meal plans gradually. It has been thought that this approach reduces the chance of patients having refeeding syndrome, a

\footnotetext{
* Correspondence: ksmith@nrifargo.com

${ }^{1}$ Neuropsychiatric Research Institute, Fargo, North Dakota, USA

Full list of author information is available at the end of the article
}

potentially fatal complication that can happen when severely underweight individuals begin to eat more. However, a gradual approach to refeeding prolongs hospitalization and causes more disruption for patients and families. Our study examined a refeeding program that started patients with AN and atypical AN on higher calorie meal plans and advanced their calories more rapidly than traditional approaches to inpatient hospital treatment. Results showed that this protocol was related to increased weight gain, but was not related to readmission or refeeding syndrome. Our findings support 
a more rapid approach to refeeding patients with AN and atypical AN in the hospital.

\section{Background}

Children and adolescents with eating disorders may require inpatient hospitalization for medical stabilization and weight regain [1-3], with Anorexia Nervosa (AN) accounting for a significant proportion of such admissions [4]. Higher rates of weight gain and higher weight status upon discharge are generally predictive of better outcomes and weight maintenance after inpatient treatment [5-7]. Despite the importance of early weight changes in long-term recovery from AN, current national standards in the U.S. recommend relatively conservative approaches to refeeding in AN (e.g., starting at 1200 calories and increasing by 200 calories every other day) $[8,9]$.

Such "start low, go slow" approaches were developed with the aim of reducing the risk of refeeding syndrome, a potentially fatal condition that may occur when nutrition is re-introduced to a severely malnourished individual. Although there are no strict definitions, refeeding syndrome is characterized by a constellation of symptoms stemming from shifts in electrolytes that may occur when refeeding malnourished individuals, which can lead to potentially lethal complications [10-12]. Early signs of refeeding syndrome include low levels of serum phosphorous, magnesium or potassium. Refeeding syndrome may cause serious clinical complications including signs of fluid shifts (e.g., edema), respiratory distress, congestive heart failure, cardiac arrhythmias, and seizures [13]. This condition is a serious risk during refeeding and has been documented in reports of AN treatment [14].

Hypophosphatemia has emerged as a clear marker of risk of refeeding syndrome, and the central role of phosphorous during refeeding is well-described [15]. The risk of refeeding hypophosphatemia also appears to be greatest for patient's presenting with more severe malnutrition $[15,16]$. Per the recent review by Garber and colleagues [16], replacing phosphorus has not been associated with adverse side effects. Despite the general consensus and recommendation to monitor and replace phosphorous during nutritional rehabilitation of malnourished patients, there are not clear guidelines for how to replace phosphorous, especially with more accelerated refeeding protocols. At our center, hospitalists replace phosphorous when it is declining, thereby possibly avoiding hypophosphatemia and preventing refeeding syndrome.

Although the "start low, go slow" approach to refeeding attempts to minimize risk of refeeding syndrome, this approach also has significant downsides, in that it incurs more cost and burden to families, and in some studies has been shown to result in initial weight loss and slower rates of weight gain while during hospitalization [17]. These findings are particularly important in light of altered metabolic processes that have been documented in AN. That is, while individuals with AN demonstrate reduced metabolic rate [18], evidence suggests that during the refeeding process they become energy inefficient due to hypermetabolism and increased dietary-induced thermogenesis (i.e., energy production caused by metabolizing food consumed) [19-21], which may persist following weight restoration [2]. This is also evidenced by the fact that individuals with $\mathrm{AN}$ require more energy per kilogram of body weight than would be expected for normal weight individuals to gain weight $[20,22]$. Therefore it is necessary to develop safe, evidence-based refeeding protocols that account for this phenomenon and provide adequate energy intake levels to accomplish ample weight regain early in treatment.

Furthermore, there is emerging evidence to suggest that hospitalized individuals with AN can tolerate more aggressive refeeding protocols, which is contrary to "start low, go slow" approaches [16, 23]. This is consistent with recently published Australian and New Zealand guidelines recommending a less conservative refeeding approach in conjunction with appropriate monitoring and supplementation of phosphorus [24]. Indeed, some studies of adolescents with AN have shown that higher calorie prescriptions and more rapid weight gain are not related to hypophosphatemia or other signs of refeeding syndrome [25-30]. Moreover, higher calories prescribed upon admission have been found to relate to shorter lengths of stay $[17,25,26]$, which has the potential to increase the cost-effectiveness of treatment and reduce burden and disruption associated with hospitalization.

Evidence also suggests that higher initial calories predict higher rates of weight gain [25], yet some research has not replicated this finding [26]. In addition, it is not clear whether higher rates of weight gain are necessarily beneficial for patients' long-term outcomes. While one study of adolescent and adult inpatients treated for AN found that higher rates of weight restoration were predictive of higher weight at 1-year follow-up [7], in another inpatient sample, more rapid weight gain was found to predict higher rates of re-hospitalization [31]. Additionally, in adolescents, some studies have found that weight status at admission and discharge was not related to clinical outcomes [32, 33].

Despite some variations between studies, a previous synthesis of studies of more rapid refeeding approaches to refeeding in AN summarized the evidence for the "start high, advance fast" approach to refeeding in AN patients (at 75-85\% of median Body Mass Index, $\% \mathrm{mBMI}$ ) in hospital as (1) beginning at $1500 \mathrm{kcal}$ or higher; (2) advancing by $250 \mathrm{kcal}$ every day or every 
other day to reach $2500-3000$ kcal by day 14 ; (3) weekly weight gain of approximately $1.5 \mathrm{~kg}$ (3.3 lbs); (4) a lack of indicators of refeeding syndrome; and (5) achieving medical stability by approximately day 14 [34]. However, a recent review of refeeding protocols by Garber and colleagues [16] revealed that only seven studies thus far have examined higher calorie meal-based protocols, leaving much unknown about specific factors that influence patients' weight gain during hospital stay and their continued weight restoration post-discharge. Furthermore, thus far only one RCT has compared different approaches to refeeding [35]. This study, which assessed young adults with a duration of illness of 3-4 years who had been previously hospitalized, compared a protocol consisting of nasogastric tube feedings plus meals to meals alone [35]. Thus, there have been no RCTs comparing different meal-based approaches to refeeding in children and adolescents during their first hospitalization. In the absence of such RCTs, retrospective studies have the potential to provide further insight into the effectiveness and acceptability of more rapid refeeding protocols.

Therefore, the present study sought to examine the safety and effectiveness of a more accelerated mealbased refeeding protocol for youth with $\mathrm{AN}$ and atypical AN who were treated on an inpatient unit. It was predicted that (1) this protocol would result in significant increases in weight status (\%mBMI) without the occurrence of refeeding syndrome; (2) higher rates of caloric change throughout treatment would be related to greater change in \%mBMI during and after hospitalization; (3) higher rates of caloric and \%mBMI change during hospital stay would not be related to an increased likelihood of re-hospitalization; and (4) higher calories prescribed upon discharge would be predictive of greater \%mBMI increase between the time of discharge and follow-up assessment.

\section{Methods}

\section{Study design and participants}

A retrospective chart review was conducted for consecutive admissions of patients (ages 22 and younger) with a primary diagnosis of $\mathrm{AN}$ or atypical $\mathrm{AN}^{1}$ who were treated in the inpatient medical stabilization unit for eating disorders at Children's Hospitals and Clinics of Minnesota from 2012 to 2015. Only first episodes of inpatient hospitalization at this facility were included in the analyses. Admission and discharge criteria are described in Table 1. Admissions were based on clinical evaluation and the requirement to meet at least one admission criteria, which were based on previously established guidelines in the U.S. $[1,3]$. Readiness for discharge was assessed by a team of hospitalists and eating disorder specialists, including psychiatrists, psychologists, and social workers. Patients were deemed ready for
Table 1 Inpatient admission and discharge criteria

\begin{tabular}{|c|c|}
\hline Admission criteria & Discharge criteria \\
\hline Weight $<75 \%$ of median BMI (\%mBMI) & Medical stabilization \\
\hline $\begin{array}{l}\text { Rapid weight loss, even if weight not } \\
<75 \%\end{array}$ & $\begin{array}{l}\text { Admission criteria are no } \\
\text { longer met }\end{array}$ \\
\hline Acute food refusal & $\begin{array}{l}\text { Adequate oral intake for } \\
\text { weight regain }\end{array}$ \\
\hline Heart rate $<50$ bpm & Cessation of vomiting \\
\hline $\begin{array}{l}\text { Orthostatic hypotension (increase in pulse } \\
\text { of }>20 \mathrm{bpm} \text { or drop in blood pressure of } \\
10-20 \mathrm{mmHg} / \mathrm{min} \text { from supine to standing) }\end{array}$ & $\begin{array}{l}\text { Has completed } 1 \text { staff led } \\
\text { practice meal and } 1-3 \\
\text { family led/individual } \\
\text { practice meals }\end{array}$ \\
\hline \multicolumn{2}{|l|}{ Blood pressure $<80 / 50$} \\
\hline \multicolumn{2}{|l|}{ Hypokalemia } \\
\hline \multicolumn{2}{|l|}{ Hypophosphatemia } \\
\hline \multicolumn{2}{|l|}{ Hypomagnesemia } \\
\hline \multicolumn{2}{|l|}{ Dehydration } \\
\hline \multicolumn{2}{|l|}{ Cardiac arrhythmia } \\
\hline \multicolumn{2}{|l|}{ Hypothermia } \\
\hline \multicolumn{2}{|l|}{ Syncope } \\
\hline \multicolumn{2}{|l|}{ Symptomatic hypoglycemia } \\
\hline \multicolumn{2}{|l|}{$\begin{array}{l}\text { Lack of improvement/worsening symptoms } \\
\text { despite outpatient treatment }\end{array}$} \\
\hline Uncontrolled vomiting or hematemesis & \\
\hline
\end{tabular}

discharge when they showed improvement in vital signs and electrolytes, had adequate oral intake for weight regain, reached at least $75 \% \mathrm{mBMI}$, had completed at least one therapist-led family meal and one family-led practice meal, and had an outpatient treatment plan in place. Participants who started outpatient treatment after discharge completed a follow-up assessment approximately 4 weeks after discharge. Data was not available for participants who did not follow-up in our facility. Participants were excluded from analyses if they received nasojejunal (NJ) or nasogastric (NG) tube feeding during treatment, as they did not follow the same refeeding protocol. No participants were excluded due to co-occurring medical or psychiatric conditions. This study was approved by the Institutional Review Board at Children's Hospitals and Clinics of Minnesota.

\section{Refeeding protocol}

The refeeding protocol consisted of three meals and two snacks (afternoon and evening). Meals and snacks were consumed in a supervised group dining room on the unit. If participants were unable to consume the food provided on their tray, they were offered a meal replacement (i.e., high-calorie liquid supplement) that provided an equivalent amount of energy. Participants were supervised by program staff for $60 \mathrm{~min}$ after meals and 30 min after snacks. In addition to meals, participants 
attended three groups per week on topics including emotional regulation, problem solving and interpersonal effectiveness/communication. Participants received additional services including music therapy, child life programming, physical therapy, and integrative medicine. Participants were typically on telemetry for up to the first $72 \mathrm{~h}$ following admission, or longer until their heart rate trends were approaching 50 beats per minute. While on telemetry, participants were required to remain on the unit. Following this, if participants were finishing meals they were allowed privileges such as going off the unit (within the hospital grounds) with parents or hospital staff. On the medical unit, bathrooms were not locked and participants were not given additional replacements if they were found to be engaging in exercise. Participants were under staff supervision for the majority of the day and into the evening, with additional support requested from parents if needed. Participants were required to be completing most meals and replacements to attend physical therapy groups, which occurred several times per week.

Most participants started on a $1500 \mathrm{kcal}$ per day regimen. Calorie increases were made in $500 \mathrm{kcal}$ increments in order to attain an inpatient rate of weight regain of $130-200 \mathrm{~g}$ per day. The program included collaborative weighing sessions twice weekly, in which progress with weight regain was discussed openly with the patient and family. The macronutrient composition of the diet consisted of 50-60 \% carbohydrate, $20-30 \%$ fat, and $15-20 \%$ protein. Fluid requirements were calculated for each participant based on his/her current weight using the Holliday-Segar Method [36]. Intravenous (IV) fluids were administered if participants exhibited significant signs of dehydration, hypotension, and/or uncontrolled vomiting, or as otherwise clinically indicated. Daily fluid intake and urine output were recorded throughout hospitalization. Standard laboratory values were taken upon admission, and most were repeated at discharge. Electrolytes, including phosphorus, were checked at least twice weekly. While there are few data on inpatient supplementation protocols during refeeding for patients with AN [37], given our accelerated meal-based refeeding protocol, phosphorus was replaced frequently with the goal of maintaining a level of $4.0 \mathrm{mg} / \mathrm{dL}$. Two formulations of phosphorus were utilized, including Neutra-Phos tablets and K-Phos Neutral tablets or packets.

\section{Data collection}

Chart reviews were conducted to extract assessments of vital signs, laboratory values, and weight status during hospitalization. Follow-up weight status was assessed at participants' 4-week follow-up in the outpatient clinic, where Family-Based Treatment (FBT) [38] or Cognitive
Behavioral Therapy-Enhanced (CBT-E) [39] were offered as first-line treatments. Re-admission to the inpatient unit within 4 weeks of discharge was documented. While it was not possible to verify whether patients were admitted to another facility, Children's is the only hospital in the region specializing in the medical stabilization of pediatric patients with eating disorders.

\section{Indicators of refeeding syndrome}

Given the difficulties of quantifying all of the symptoms that comprise refeeding syndrome, it has been recommended that refeeding hypophosphatemia (i.e., serum phosphorus level below $3 \mathrm{mg} / \mathrm{dL}$ ) be considered as an indicator of risk of refeeding syndrome [15]. Consistent with this guideline, this study evaluated risk of refeeding syndrome by assessing refeeding hypophosphatemia at any time during hospitalization. Chart reviews were also conducted for all participants to assess for the presence of full refeeding syndrome. In addition, hospitalists working in this unit consider the occurrence of multiple electrolyte imbalances during refeeding, specifically hypophosphatemia, hypokalemia, and hypomagnesemia, to be indicative of a high likelihood of full refeeding syndrome. Given this practice, in order to quantitatively operationalize a high likelihood of full refeeding syndrome, we also evaluated whether any participants evidenced all three of these electrolyte abnormalities during their treatment (but not necessarily concurrently).

\section{Statistical methods}

Weight status was calculated based on the participant's age, height, sex, and corresponding Centers for Disease Control and Prevention (CDC) 50th percentile BMI-forage [40], which is the expected median BMI among normally developing adolescents of similar age, sex, and height. Participant's weight status was expressed as a percentage of this value (\%mBMI). The Estimated Energy Requirement (EER) of each participant was calculated retrospectively [41], which approximates the energy intake required to maintain weight in a healthy individual of similar age, sex, weight, and height at a low activity level (Daily PAL: 1.0). Given that the EER underestimates the energy needs of those with AN, EER was calculated based on the BMI corresponding to the CDC median BMI for age and sex, which is in line with previous research [25].

Caloric changes were calculated both as raw values and as percentages of participants' EER in order to assess the degree of energy surplus that was provided relative to their EER. The rate of caloric change during treatment was calculated by dividing the change in calories between admission and discharge by their length of stay, expressed in $\mathrm{kcal} /$ day. 
Growth curve models were used to assess the nature of change in \%mBMI over time, and predictors of $\% \mathrm{mBMI}$ change were subsequently added to these models using Hierarchical Linear Modeling (HLM) [42]. First, unconditional growth curve models investigated whether there was a linear or nonlinear change over time, which included both linear $\left(\pi_{1 i}\right)$ and quadratic $\left(\pi_{2 i}\right)$ growth parameters, e.g.:

\section{Level-1 Model}

$$
\begin{aligned}
\% m B M I_{t i}= & \pi_{0 i}+\pi_{1 i} *\left(\text { Time }_{t i}\right)+\pi_{2 i} *\left(\text { Time }_{t i}\right)^{2} \\
& +e_{t i}
\end{aligned}
$$

\section{Level-2 Model}

$$
\begin{aligned}
& \pi_{0 i}=\beta_{00}+r_{0 i} \\
& \pi_{1 i}=\beta_{10}+r_{1 i} \\
& \pi_{2 i}=\beta_{20}+r_{2 i}
\end{aligned}
$$

Given that it is likely that the rate of weight gain varied between the time of hospitalization and posthospitalization, a piecewise linear growth model also estimated growth rates separately during hospitalization (admission to discharge) and post-hospitalization (discharge to follow-up). Time-varying (level 1) and personlevel (level 2) predictors were subsequently added to models to assess the relationship between the rate of caloric change and \%mBMI change during and after hospitalization. In these conditional models, the rate of caloric change was entered at level 1, while age and EER were entered at level 2 . In the model assessing posthospitalization \%mBMI, discharge calorie level was also entered as a predictor. A Bernoulli model assessed whether higher rates of caloric and \%mBMI change were related to an increased likelihood of re-hospitalization after discharge (binary outcome).

To evaluate the safety of the refeeding protocol, the number and percentage of participants who evidenced refeeding hypophosphatemia was assessed, as well as whether any cases met the aforementioned criteria for refeeding syndrome. A binary logistic regression model also assessed predictors (i.e., admission \%mBMI, age, and rates of \%mBMI and caloric changes) of hypophosphatemia at any point during treatment. Analyses were conducted using SPSS 24 [43] and HLM 7.01 [44].

\section{Results}

\section{Sample characteristics}

Table 2 displays characteristics of the sample. Eleven participants were excluded from analyses because they had NG/NJ tubes at some point during their treatment, which resulted in a total sample of 129. Participants were predominately female $(94.6 \%)$ and ranged in age from 10 to $22(M=15.84, S D=2.37)$. Most identified as
non-Hispanic Caucasian (89.1\%), and the remaining as Asian American (3.9\%), African American (1.6 \%), Hispanic/Latino (1.6\%), American Indian/Alaskan $0.8 \%$ ), and multiracial (0.8\%). Three participants $(2.3 \%)$ did not report this information. There were five participants for which admission \%mBMI could not be calculated because their age exceeded the maximum age specified in CDC BMI-for-age charts. These participants were excluded from analyses involving \%mBMI estimations. Of the total sample, $113(87.6 \%)$ patients had $\mathrm{AN}$, and 16 (12.4\%) had atypical AN.

Of the 129 participants, $12(9.3 \%)$ were re-admitted within 4 weeks, and 89 (69.0 \%) completed the 4-week follow-up. Using an adjusted significance level for multiple comparisons $(p<.01)$, participants who did and did not complete follow up did not evidence significant differences in admission \%mBMI $(t[122]=1.41, p=.162)$, discharge \%mBMI $(t[122]=1.37, p=.175)$, length of stay $(t[127]=.511, p=.610)$, rate of caloric change $(t[127]=$ $1.80, p=.074)$, age $(t[127]=2.11, p=.036)$, or proportion of males compared to females $\left(\chi^{2}[1]=1.97, p=.160\right)$.

\section{Safety and effectiveness}

Laboratory values upon admission, during treatment, and at discharge are displayed in Table 3. Subsequent to admission, rates of hypophosphatemia, hypocalcemia, hyponatremia, hypokalemia, and hypomagnesaemia were $47.3 \%(n=61), 43.4 \%(n=56), 18.6 \%(n=24), 12.4 \%$ $(n=16)$, and $1.6 \%(n=2)$, respectively. a Participant age, sex, rates of caloric or \%mBMI change, or admission $\%$ mBMI were not significant predictors of hypophosphatemia during hospitalization (adjusting for length of stay). No participants evidenced hypophosphatemia at discharge, although 100 patients $(77.5 \%)$ were prescribed phosphorus supplements at discharge. Of the patients for whom the discharge phosphorus dose was available $(n=98)$, the mean dose was $1012.76 \mathrm{mg}(S D=$ 611.71; range: 250-3000 mg). Despite the significant number of participants evidencing risk for refeeding syndrome during treatment, both chart review and quantitative assessment of refeeding criteria (i.e. hypophosphatemia, hypokalemia, and hypomagnesemia, each at some point during hospitalization) did not identify any cases of full refeeding syndrome.

Other medications prescribed to patients are shown in Table 4. With respect to psychotropic medications, a substantial proportion of patients $(n=57 ; 44.2 \%)$ were prescribed antidepressants (i.e., fluoxetine, amitriptyline, citalopram, escitalopram), while a smaller number were prescribed atypical antipsychotic medication $(n=6 ; 4.7 \%)$.

\section{Changes in calories}

The majority of patients (89.9\%) were started on $1500 \mathrm{kcal}$ meal plans upon admission, and on average, 
Table 2 Descriptive statistics

\begin{tabular}{|c|c|c|c|c|c|}
\hline & $N$ & Min & Max & $M$ & SD \\
\hline Length of stay (days) & 129 & 0.88 & 40.01 & 14.98 & 7.51 \\
\hline Participant age & 129 & 10.04 & 22.94 & 15.84 & 2.37 \\
\hline Admission initial calories & 129 & 1500.00 & 3000.00 & 1585.27 & 280.37 \\
\hline Day 14 prescribed calories & 71 & 1500.00 & 5000.00 & 3626.17 & 644.68 \\
\hline Discharge calories & 129 & 1500.00 & 5000.00 & 3771.32 & 829.17 \\
\hline Admission initial calories \% of EER & 129 & 29.02 & 131.32 & 64.52 & 14.34 \\
\hline Discharge calories \% of EER & 129 & 59.00 & 230.08 & 152.81 & 37.43 \\
\hline Admission to discharge calorie change & 129 & 0.00 & 3500.00 & 2186.05 & 875.16 \\
\hline Calorie change (admission to discharge) \% of EER & 129 & 0.00 & 238.61 & 130.21 & 52.46 \\
\hline Admission \%mBMl & 124 & 61.51 & 110.64 & 79.40 & 8.47 \\
\hline Discharge \%mBMl & 124 & 64.40 & 111.18 & 85.54 & 8.11 \\
\hline Four-week follow-up \%mBMI & 74 & 72.07 & 113.70 & 89.84 & 7.92 \\
\hline Admission BMl & 129 & 11.90 & 20.60 & 15.86 & 1.81 \\
\hline Discharge BMI & 129 & 13.70 & 21.40 & 17.12 & 1.62 \\
\hline Days between discharge and follow-up & 83 & 21.00 & 44.00 & 26.07 & 5.59 \\
\hline Days on telemetry & 127 & 0.00 & 14.00 & 2.84 & 2.18 \\
\hline Days administered (IV) fluids & 11 & 1.00 & 2.00 & 1.18 & 0.40 \\
\hline Fluid balance day $1(\mathrm{~mL})$ & 127 & -4070.00 & 2818.00 & -431.59 & 945.97 \\
\hline Fluid balance day $2(\mathrm{~mL})$ & 126 & -6005.00 & 1570.00 & -733.80 & 1079.87 \\
\hline Fluid balance day $3(\mathrm{~mL})$ & 122 & -5125.00 & 1920.00 & -480.36 & 1015.64 \\
\hline \multicolumn{6}{|l|}{ Rate of calorie change (kcal/day) } \\
\hline Admission to discharge & 129 & 0.00 & 539.12 & 163.87 & 72.65 \\
\hline Week 1 (Day 1-7) & 116 & -14.29 & 457.14 & 185.16 & 82.57 \\
\hline Week 2 (Day 7-14) & 70 & -123.81 & 300.00 & 120.48 & 76.66 \\
\hline Week 3 (Day 14-21) & 28 & -250.00 & 200.00 & 1.33 & 113.07 \\
\hline Week 4 (Day 21-28) & 9 & -100.00 & 392.86 & 80.95 & 136.59 \\
\hline
\end{tabular}

$\% m B M I$ Percentage of median BMI, EER Estimated Energy Requirement, BMI Body Mass Index

$\% \mathrm{mBMI}$ was not calculated for individuals over 20 years of age $(n=6)$ due to upper limits of the Center for Disease Control (CDC) BMI-for-age percentile charts Fluid balance was calculated as total input minus total output

participants increased to over $3600 \mathrm{kcal}$ by day 14 . The mean prescribed calorie level at discharge (i.e., approximately $3771 \mathrm{kcal}$ ) represented over $150 \%$ of participants' EER (Table 2). As shown in Table 2, there was a mean increase of $163.87 \mathrm{kcal}$ per day.

\section{Changes in weight and \%mBMI}

Participants evidenced a mean weekly weight gain of $1.39 \mathrm{~kg}(S D=1.49)$. Table 5 displays results of growth curve analyses estimating changes in \%mBMI. As displayed in Fig. 1, results indicated an average linear increase of 0.43 in \%mBMI per day from the time of admission to follow-up, with the quadratic slope estimate indicating a significant deceleration over time (Table 5, Model 1). However, when considering only the time during hospitalization, there was not significant acceleration or deceleration in growth (Model 2), suggesting a linear change during hospitalization.
The piecewise growth model (Model 3) indicated that participants increased an average of 0.38 per day in \%mBMI during hospitalization compared to 0.09 per day between the time of discharge and follow-up (Fig. 2), with both slopes being significantly greater than zero.

\section{Predictors of \%mBMI}

As indicated by results of conditional models [4, 5], younger participants evidenced lower initial \%mBMI. Higher rates of caloric increases were associated with greater increases in \%mBMI during but not after hospitalization (Models 4-5). The number of calories prescribed at discharge was not related to change in \%mBMI post-hospitalization (Model 5). A Bernoulli model (Model 6) estimating the probability of rehospitalization after discharge found that neither the rate of caloric change (OR: .99; CI: .98-1.00) nor rate of \%mBMI change (OR: .22; CI: .01-6.06) was associated 
Table 3 Laboratory values at admission, during treatment, and discharge

\begin{tabular}{|c|c|c|c|c|c|c|c|c|c|c|c|c|c|c|c|c|c|c|c|}
\hline \multirow[b]{3}{*}{ Test } & \multirow[b]{3}{*}{ Reference range } & \multicolumn{7}{|c|}{ Admission } & \multicolumn{4}{|c|}{ During Treatment } & \multicolumn{7}{|c|}{ Discharge } \\
\hline & & \multirow[b]{2}{*}{$N$} & \multirow[b]{2}{*}{ M } & \multirow[b]{2}{*}{$S D$} & \multicolumn{2}{|c|}{$\begin{array}{l}\text { Below } \\
\text { reference } \\
\text { range }\end{array}$} & \multicolumn{2}{|c|}{$\begin{array}{l}\text { Above } \\
\text { reference } \\
\text { range }\end{array}$} & \multicolumn{2}{|c|}{$\begin{array}{l}\text { Below } \\
\text { reference } \\
\text { range }\end{array}$} & \multicolumn{2}{|c|}{$\begin{array}{l}\text { Above } \\
\text { reference } \\
\text { range }\end{array}$} & \multirow[b]{2}{*}{$N$} & \multirow[b]{2}{*}{ M } & \multirow[b]{2}{*}{$S D$} & \multicolumn{2}{|c|}{$\begin{array}{l}\text { Below } \\
\text { reference } \\
\text { range }\end{array}$} & \multicolumn{2}{|c|}{$\begin{array}{l}\text { Above } \\
\text { reference } \\
\text { range }\end{array}$} \\
\hline & & & & & $N$ & $\%$ & $N$ & $\%$ & $N$ & $\%$ & $N$ & $\%$ & & & & $N$ & $\%$ & $N$ & $\%$ \\
\hline Sodium & $137-147 \mathrm{mEq} / \mathrm{L}$ & 129 & 139.39 & 2.49 & 15 & 11.63 & 0 & 0 & 24 & 18.60 & 1 & 0.78 & 126 & 140.21 & 1.77 & 0 & 0 & 0 & 0 \\
\hline \multirow[t]{2}{*}{ BUN } & Age < 18: 9-18 mg/dL & 129 & 14.57 & 5.62 & 13 & 10.08 & 24 & 18.60 & 28 & 21.71 & 40 & 31.01 & 126 & 14.36 & 4.13 & 7 & 5.43 & 14 & 10.85 \\
\hline & Age >18: 8-22 mg/dL & & & & & & & & & & & & & & & & & & \\
\hline Potassium & $3.5-5.3 \mathrm{mEq} / \mathrm{L}$ & 129 & 3.87 & 0.37 & 11 & 8.53 & 0 & 0 & 16 & 12.40 & 2 & 1.55 & 126 & 4.15 & 0.30 & 0 & 0 & 0 & 0 \\
\hline Glucose & $70-100$ mg/dL & 129 & 81.62 & 18.64 & 34 & 26.36 & 20 & 15.50 & 54 & 41.86 & 11 & 8.53 & 126 & 82.11 & 9.60 & 10 & 7.75 & 5 & 3.88 \\
\hline Calcium & $8.6-10.6 \mathrm{mg} / \mathrm{dL}$ & 129 & 6.65 & 2.30 & 69 & 53.49 & 0 & 0 & 56 & 43.41 & 0 & 0.00 & 126 & 6.61 & 2.28 & 72 & 55.81 & 0 & 0 \\
\hline Phosphorus & $3.0-5.5 \mathrm{mg} / \mathrm{dL}$ & 129 & 3.63 & 0.58 & 13 & 10.07 & 0 & 0 & 61 & 47.28 & 9 & 6.98 & 125 & 4.34 & 0.57 & 0 & 0 & 3 & 2.33 \\
\hline Magnesium & $1.5-2.5 \mathrm{mg} / \mathrm{dL}$ & 126 & 1.87 & 0.23 & 2 & 1.55 & 0 & 0 & 2 & 1.55 & 1 & 0.78 & 126 & 1.84 & 0.23 & 1 & 0.78 & 0 & 0 \\
\hline Chloride & 98-106 mEq/L & 129 & 103.84 & 3.16 & 5 & 3.88 & 24 & 19 & 6 & 4.65 & 74 & 57.36 & 126 & 105.10 & 2.46 & 0 & 0 & 31 & 24.03 \\
\hline Creatinine & $0.38-0.99 \mathrm{mg} / \mathrm{dL}$ & 129 & 0.77 & 0.16 & 0 & 0 & 15 & 11.63 & 0 & 0 & 14 & 10.85 & 125 & 0.68 & 0.14 & 0 & 0 & 4 & 3.10 \\
\hline $\mathrm{AST}^{\mathrm{a}}$ & $10-41 \mathrm{U} / \mathrm{L}$ & 110 & 27.70 & 40.37 & 1 & 0.78 & 9 & 6.98 & & & & & & & & & & & \\
\hline $\mathrm{ALT}^{\mathrm{a}}$ & $30-65 \mathrm{U} / \mathrm{L}$ & 110 & 43.32 & 68.15 & 45 & 34.88 & 9 & 6.98 & & & & & & & & & & & \\
\hline Albumin ${ }^{a}$ & $3.5-5.0 \mathrm{~g} / \mathrm{dL}$ & 119 & 4.30 & 0.46 & 4 & 3.10 & 5 & 3.88 & & & & & & & & & & & \\
\hline Protein $^{a}$ & $6.0-8.0 \mathrm{~g} / \mathrm{dL}$ & 110 & 7.43 & 0.61 & 1 & 0.78 & 15 & 11.63 & & & & & & & & & & & \\
\hline$W B C^{a}$ & $4.5-13 \mathrm{~K} / \mathrm{LL}$ & 115 & 5.45 & 1.54 & 32 & 24.81 & 0 & 0 & & & & & & & & & & & \\
\hline $\mathrm{TSH}^{\mathrm{a}}$ & $.5-4.8 \mathrm{ulU} / \mathrm{mL}$ & 103 & 1.81 & 1.49 & 9 & 6.98 & 5 & 3.88 & & & & & & & & & & & \\
\hline Prealbumin ${ }^{a}$ & $21.2-42.4 \mathrm{mg} / \mathrm{dL}$ & 37 & 22.08 & 5.36 & 17 & 13.18 & 0 & 0 & & & & & & & & & & & \\
\hline
\end{tabular}

${ }^{\mathrm{a}}$ Not repeated during treatment or at discharge

with re-hospitalization. Exploratory analyses revealed that the rate of caloric change was not correlated with the number of meal replacements administered (Spearman's $\rho=-.11, p=.345)$ or number of incidences of food refusal $(\rho=.13, p=.492)$ during hospitalization.

Notably, three participants (2.33\%) evidenced decreases in \%mBMI between admission and discharge. Compared to the rest of the sample, these individuals had significantly shorter lengths of stay $(M=1.89$ days, $S D=1.03, t[120]=-15.04, p=<.001$ ), lower calories (in relationship to their EER) prescribed at discharge

Table 4 Summary of medications prescribed to patients

\begin{tabular}{lll}
\hline Medication & $n$ & $\%$ \\
\hline Hydroxyzine & 64 & 49.61 \\
Polyethylene glycol & 62 & 48.06 \\
Fluoxetine & 53 & 41.09 \\
Ondansetron & 22 & 17.05 \\
Lansoprazole & 15 & 11.63 \\
Olanzapine & 6 & 4.65 \\
Amitriptyline & 2 & 1.55 \\
Citalopram & 1 & 0.78 \\
Erythromycin & 1 & 0.78 \\
Escitalopram & 1 & 0.78 \\
\hline
\end{tabular}

$(t[120]=-3.26, p=.001)$, yet the rate of caloric change did not differ significantly between groups $(t[120]=.84$, $p=.491)$. Chart review of these cases found that these participants left prematurely against recommendations of the treatment team.

\section{Discussion}

The present study aimed to assess the safety and effectiveness of a more accelerated refeeding protocol for hospitalized youth with AN and atypical AN. This protocol was largely consistent with the "start high, advance fast" approach [34], as evidenced in this sample by (1) a mean weight gain of approximately $1.4 \mathrm{~kg}$ per week; (2) medical stabilization for discharge within 15 days; and (3) a mean caloric increase of $164 \mathrm{kcal}$ per day, which exceeded $3600 \mathrm{kcal}$ by day 14. In addition, this protocol was able to be implemented with exclusively oral intake, with a small number of participants receiving NJ/NG tube feedings $(n=11)$ who were excluded from analyses.

Consistent with previous literature [16], youth with AN and atypical AN were able to tolerate more aggressive refeeding during hospitalization without any occurrences of refeeding syndrome. However, there was a significant risk of refeeding syndrome during hospitalization, as evidenced by hypophosphatemia in 
Table 5 Summary of growth curve model results

\begin{tabular}{|c|c|c|c|c|c|}
\hline Dependent variable & Independent variables & Estimate & SE & $t$ & $p$ \\
\hline 1. \%mBMl change & Intercept & 79.36 & 0.78 & 101.17 & $<0.001$ \\
\hline from admission & Time & 0.43 & 0.02 & 17.43 & $<0.001$ \\
\hline to follow-up & Time $^{2}$ & $<0.01$ & $<0.01$ & -7.55 & $<0.001$ \\
\hline 2. \%mBMl change & Intercept & 79.39 & 0.75 & 105.18 & $<0.001$ \\
\hline during & Hospital time & 0.37 & 0.09 & 4.38 & $<0.001$ \\
\hline hospitalization & Hospital time ${ }^{2}$ & $<0.01$ & $<0.01$ & 0.59 & 0.558 \\
\hline 3. \%mBMl change during & Intercept & 79.40 & 0.79 & 100.48 & $<0.001$ \\
\hline hospitalization vs. & Hospitalization time & 0.38 & 0.02 & 17.85 & $<0.001$ \\
\hline post-hospitalization & Post-hospitalization time & 0.09 & 0.01 & 6.56 & $<0.001$ \\
\hline \multicolumn{6}{|l|}{ Conditional models } \\
\hline 4. \%mBMl change & Intercept & 79.28 & 1.02 & 77.66 & $<0.001$ \\
\hline during & Age & -0.99 & 0.36 & -2.72 & 0.007 \\
\hline \multirow[t]{4}{*}{ hospitalization } & Calorie rate & 0.04 & 0.01 & 3.03 & 0.003 \\
\hline & Age & -0.01 & 0.01 & -2.08 & 0.038 \\
\hline & EER & $<0.01$ & $<0.01$ & 0.48 & 0.632 \\
\hline & Time (hospitalization) & 0.29 & 0.01 & 54.7 & $<0.001$ \\
\hline 5. \%mBMl change & Intercept & 87.37 & 2.80 & 31.25 & $<0.001$ \\
\hline \multirow[t]{8}{*}{ post-hospitalization } & Age & -1.55 & 0.52 & -2.97 & 0.004 \\
\hline & Calorie rate & $<0.01$ & $<0.01$ & -0.10 & 0.924 \\
\hline & Age & $<0.01$ & 0.01 & -2.08 & 0.041 \\
\hline & EER & $<0.01$ & $<0.01$ & 0.69 & 0.493 \\
\hline & Discharge calories & $<0.01$ & $<0.01$ & -1.67 & 0.099 \\
\hline & Age & $<0.01$ & $<0.01$ & 1.49 & 0.141 \\
\hline & EER & $<0.01$ & $<0.01$ & -1.15 & 0.255 \\
\hline & Time (post-hospitalization) & 0.08 & 0.11 & 0.70 & 0.489 \\
\hline 6. Probability of re- & Intercept & -2.33 & 0.35 & -6.65 & $<0.001$ \\
\hline \multirow[t]{8}{*}{ admission } & Age & -0.19 & 0.17 & -1.10 & 0.273 \\
\hline & Discharge \%mBMl & 0.05 & 0.04 & 1.05 & 0.296 \\
\hline & Age & -0.01 & 0.02 & -0.53 & 0.597 \\
\hline & $\%$ mBMI rate & -1.50 & 1.67 & -0.90 & 0.371 \\
\hline & Age & 2.03 & 1.05 & 1.94 & 0.055 \\
\hline & Calorie rate & -0.01 & 0.01 & -1.70 & 0.092 \\
\hline & Age & $<0.01$ & $<0.01$ & -0.75 & 0.452 \\
\hline & EER & $<0.01$ & $<0.01$ & -1.23 & 0.222 \\
\hline
\end{tabular}

Time was scaled for hospitalization days or post-hospitalization days according to the specified model

EER estimated energy requirement, \%mBMI percentage of median $\mathrm{BMI}$

$47.3 \%$ of participants. Nevertheless, while the proportion of participants who evidenced hypophosphatemia in this study was comparable to that observed in previous research [25], it appears more participants were prescribed phosphorus supplements in this study compared to others $[25,28]$. It is important to note that electrolytes were monitored closely during treatment with aggressive phosphorus supplementation, which likely helped to prevent progression to refeeding syndrome.
In line with Golden and colleagues [26], hypophosphatemia was unrelated to caloric or \%mBMI changes. Admission weight was not related to hypophosphatemia in this study, which is contrary to previous research demonstrating a relationship between the degree of malnutrition and hypophosphatemia $[15,45]$. It is not clear why this was observed in the present sample, as participants evidenced similar admission \%mBMI compared to other research [25]. One possible explanation is that 


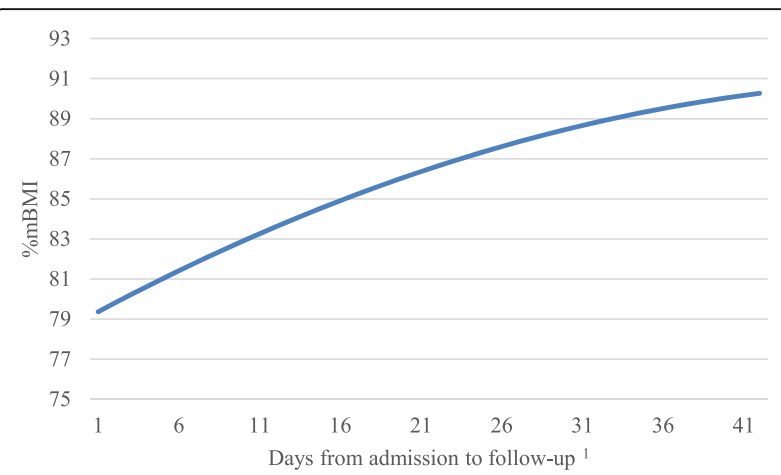

Fig. 1 Displays the curvilinear change in \%mBMl between admission and follow-up. ${ }^{1}$ The mean length of stay (14.98 days) and mean days between discharge and follow-up (26.07 days) were summed to determine the range of values for the $x$-axis. Follow-up data points represented only patients who completed this assessment

preventative phosphorus supplementation for all patients who evidenced low or declining phosphorus values negated differences between patients with lower and higher $\% \mathrm{mBMI}$ at admission. Furthermore, as noted by the recent position statement of the Society for Adolescent Health and Medicine [15], refeeding hypophosphatemia may present at any weight after a period of malnutrition. The present study also did not assess the degree of weight loss prior to hospitalization, which has been shown to be predictive of hypophosphatemia during treatment [26]. This may have occurred in the present sample, and it would be useful for future studies to explore this risk factor. Thus, while the current study did not demonstrate a relationship between hypophosphatemia and $\% \mathrm{mBMI}$ at admission, given the prophylactic phosphorus supplementation, findings do not discount the need for medical providers to be cautious of the risk of hypophosphatemia in severely malnourished patients [15].

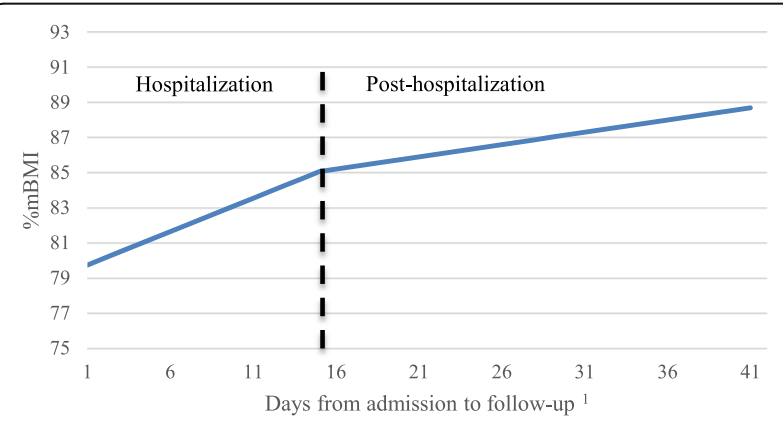

Fig. 2 Changes in mean percentage of median BMI (\%mBMI) during and after hospitalization. Figure 2 displays the results of the piecewise growth model illustrating the differing rates of change in \%mBMl during and after hospitalization. ${ }^{1}$ The mean length of stay (14.98 days) and mean days between discharge and follow-up (26.07) were summed to determine the range of values for the x-axis. Follow-up data points represented only patients who completed this assessment
This was one of few studies to include a posthospitalization assessment of a more aggressive refeeding protocol. Both caloric and \%mBMI changes were greatest early on during hospitalization; not surprisingly, $\% \mathrm{mBMI}$ change was greater during hospitalization compared to post-hospitalization. Nevertheless, participants continued to demonstrate significant increases in $\%$ mBMI after discharge, during which time they were engaged in outpatient therapies that supported continued weight gain. While previous studies have not specifically assessed the rate of caloric change, the present results suggested that higher rates of caloric increases were related to greater increases in \%mBMI during but not after hospitalization. Thus, in addition to the existing support for starting at higher initial caloric levels $[25,26]$, these findings describe the specific relationship between rate of caloric change and degree of weight change during hospitalization. It is also notable that the rate of caloric change or number of calories prescribed at discharge were not predictive of post-hospitalization changes in \%mBMI. Taken together, it may be that other factors (e.g., outpatient treatment) are more influential in the rate of weight gain post-hospitalization. Of note, both of the outpatient treatments (i.e., FBT and CBT-E) were structured evidenced-based protocols that have demonstrated effectiveness in facilitating weight gain in $\mathrm{AN}$, and these specific treatments may have contributed to the observed posthospitalization weight gain, which is consist with previous research [32]. Thus, it would be beneficial for future study to assess additional variables post-hospitalization that may facilitate early response and weight gain, which has been shown to predict long-term outcome in AN [46].

As hypothesized, higher rates of caloric and \%mBMI increases were not related to an increased likelihood of re-hospitalization after discharge. These results replicate previous findings $[25,26]$, and may suggest that implementation of more accelerated refeeding protocols could result in shorter hospital stays and reduce cost and burden to patients and families. Furthermore, there is evidence to show that shorter lengths of stay followed by outpatient treatment are not detrimental to long-term physical or psychological outcomes in AN [32]. This is important to consider in light of the rising trend of residential treatments for eating disorders [47] and high cost of inpatient hospitalization $[4,48]$. Brief inpatient medical stabilization in conjunction with evidence-based outpatient care may prove vastly more cost-effective and efficacious in outcome compared to prolonged inpatient and residential treatments, though further research is necessary to demonstrate these effects.

\section{Limitations}

There were several limitations to this study. There was not a lower-calorie comparison group, which would have 
allowed for comparisons in \%mBMI change and incidence of hypophosphatemia. The timing of caloric increases was not possible to assess, which would be helpful for future studies to address. The sample included only the first inpatient admission for youth with $\mathrm{AN}$, and it is unclear whether this protocol would be similarly effective for other diagnoses, adults, or for more chronically ill patients. This study did not include measures of psychological symptoms that could assess changes in distress; however, it is notable there was not a significant relationship between caloric rate and meal replacements or food refusal, which suggests that higher caloric increases were tolerated. Re-admission beyond 4 weeks after discharge was not assessed; while there were no significant differences in many characteristics between those who completed the follow-up assessment and those who did not, it is unclear whether the latter group evidenced similar improvement in \%mBMI after discharge. Participants who completed the follow-up assessment were also engaged in structured therapies, which makes it difficult to distinguish effects of the refeeding protocol compared to those of posthospitalization treatment. Moreover, the types of posthospitalization treatment (i.e., FBT vs. CBT-E) were not assessed as a potential moderators of post-hospital weight gain or re-admission, and future research is needed to assess whether these outcomes vary according to treatment type.

\section{Conclusions}

Despite the aforementioned limitations, this study demonstrated youth with AN and atypical AN can tolerate more accelerated oral refeeding protocols without incurring refeeding syndrome. In particular, this study adds to the literature by describing the specific relationship between of the rate of caloric advances and weight changes during hospitalization. Although results lend preliminary support for more rapid caloric advances, additional research is necessary to assess long-term outcomes of such approaches, as well as investigate whether such approaches are feasible in settings outside the hospital. However, these results also underscore the importance of vigilant monitoring of indicators of refeeding syndrome, and treatment providers must be prepared to intervene with electrolyte correction. In sum, results suggest that the "starting higher, advancing faster" approach can possibly facilitate more cost-effective treatment, yet findings also underscore the importance of having available and accessible evidence-based outpatient treatments. Continued work is also needed to standardize the implementation of "starting higher, advancing faster" approaches for broader dissemination.

\section{Endnotes}

${ }^{1}$ Atypical AN was defined as \%mBMI $>89 \%$. For participants for whom \%mBMI could not be calculated because their age exceeded the maximum age specified in CDC BMI-for-age charts, atypical AN was defined as $\mathrm{BMI}>17.5$.

\section{Acknowledgements \\ The authors thank Ross Crosby, PhD, for his assistance with statistical consultation and review of this manuscript. \\ Funding \\ Funding for the research reported was provided by Children's Hospitals and Clinics of Minnesota.}

\section{Availability of data and material}

The datasets supporting the conclusions of this article are available in the FigShare repository: https://figshare.com/s/c21e4beab9d3450cb886; https:// figshare.com/s/925dba18795174166438.

\section{Authors' contributions}

KS took primary responsibility for the manuscript, including reviewing of relevant literature, developing specific hypotheses and analytic approaches, conducting analyses, interpreting results, and drafting the paper for publication. JL, BB, JC, AL, EL, RJ, SE, and $A B$ assisted with data collection and some editing of the manuscript. DLG and JL contributed to study conceptualization, protocol design, and critical review of the manuscript. All authors read and approved the manuscript.

\section{Competing interests}

The authors declare that they have no competing interests.

\section{Consent for publication}

Not applicable (this project did not report individual data).

\section{Ethics approval and consent to participate}

This project was approved by the Institutional Review Board at Children's Hospitals and Clinics of Minnesota.

\section{Author details}

${ }^{1}$ Neuropsychiatric Research Institute, Fargo, North Dakota, USA. ${ }^{2}$ Children's Hospitals and Clinics of Minnesota, Minneapolis, Minnesota, USA. ${ }^{3}$ The Children's Hospital of Philadelphia, Philadelphia, Pennsylvania, USA. ${ }^{4}$ Fairview Health Services, Minneapolis, Minnesota, USA. ${ }^{5}$ Drexel University, Philadelphia, Pennsylvania, USA. ${ }^{6}$ Department of Psychiatry, Stanford University School of Medicine, Stanford, California, USA. ${ }^{7}$ University of California, San Francisco Department of Psychiatry, San Francisco, California, USA.

Received: 14 July 2016 Accepted: 25 October 2016

Published online: 19 December 2016

\section{References}

1. Golden NH, Katzman DK, Kreipe RE, Stevens SL, Sawyer SM, Rees J, et al. Eating disorders in adolescents: position paper of the Society for Adolescent Medicine. J Adolesc Health. 2003;33(6):496-503.

2. Campbell K, Peebles R. Eating disorders in children and adolescents: state of the art review. Pediatrics. 2014;134(3):582-92.

3. Lock J, La Via MC. Practice parameter for the assessment and treatment of children and adolescents with eating disorders. J Am Acad Child Adolesc Psychiatry. 2015;54(5):412-25

4. Zhao Y, Encinosa W. An Update on Hospitalizations for Eating Disorders, 1999 to 2009: Statistical Brief \#120. Rockville: Healthcare Cost and Utilization Project (HCUP) Statistical Briefs; 2011.

5. Baran SA, Weltzin TE, Kaye WH. Low discharge weight and outcome in anorexia nervosa. Am J Psychiatry. 1995;152(7):1070-2.

6. Lock J, Litt I. What predicts maintenance of weight for adolescents medically hospitalized for anorexia nervosa? Eat Disord. 2003;11(1):1-7.

7. Lund BC, Hernandez ER, Yates WR, Mitchell JR, McKee PA, Johnson CL. Rate of inpatient weight restoration predicts outcome in anorexia nervosa. Int J Eat Disord. 2009;42(4):301-5. 
8. American Dietetic Association. Position of the American Dietetic Association: Nutrition intervention in the treatment of anorexia nervosa, bulimia nervosa, and other eating disorders. J Am Diet Assoc. 2006;106(12):2073-82.

9. American Psychiatric Association. Treatment of patients with eating disorders,third edition. American Psychiatric Association. Am J Psychiatry. 2006;163(7 Suppl):4-54.

10. Crook MA, Hally $\mathrm{V}$, Panteli JV. The importance of the refeeding syndrome. Nutrition. 2001;17(7-8):632-7.

11. Mehanna HM, Moledina J, Travis J. Refeeding syndrome: what it is, and how to prevent and treat it. BMJ. 2008;336(7659):1495-8.

12. Khan LU, Ahmed J, Khan S, Macfie J. Refeeding syndrome: a literature review. Gastroenterol Res Pract. 2011;2011:10.1155/2011/410971. Epub 2010 Aug 25.

13. Katzman DK. Medical complications in adolescents with anorexia nervosa: a review of the literature. Int J Eat Disord. 2005;37(S1):S52-9.

14. Kohn MR, Golden NH, Shenker IR. Cardiac arrest and delirium: presentations of the refeeding syndrome in severely malnourished adolescents with anorexia nervosa. J Adolesc Health. 1998;22(3):239-43.

15. Society for Adolescent Health and Medicine. Refeeding hypophosphatemia in hospitalized adolescents with anorexia nervosa: a position statement of the Society for Adolescent Health and Medicine. J Adolesc Health. 2014; 55(3):455-7.

16. Garber AK, Sawyer SM, Golden NH, Guarda AS, Katzman DK, Kohn MR, et al. A systematic review of approaches to refeeding in patients with anorexia nervosa. Int J Eat Disord. 2016;49(3):293-310.

17. Garber AK, Michihata N, Hetnal K, Shafer MA, Moscicki AB. A prospective examination of weight gain in hospitalized adolescents with anorexia nervosa on a recommended refeeding protocol. J Adolesc Health. 2012; 50(1):24-9.

18. Kosmiski L, Schmiege SJ, Mascolo M, Gaudiani J, Mehler PS. Chronic starvation secondary to anorexia nervosa is associated with an adaptive suppression of resting energy expenditure. J Clin Endocrinol Metabol. 2013; 99(3):908-14.

19. Marzola E, Nasser JA, Hashim SA, Shih PA, Kaye WH. Nutritional rehabilitation in anorexia nervosa: review of the literature and implications for treatment. BMC Psychiatry. 2013;13:290. 244X-13-290.

20. Van Wymelbeke V, Brondel L, Marcel Brun J, Rigaud D. Factors associated with the increase in resting energy expenditure during refeeding in malnourished anorexia nervosa patients. Am J Clin Nutr. 2004;80(6):1469-77.

21. Mehler PS, Winkelman AB, Andersen DM, Gaudiani JL. Nutritional rehabilitation: practical guidelines for refeeding the anorectic patient. J Nutr Metab. 2010;2010:10.1155/2010/625782. Epub 2010 Feb 7.

22. Obarzanek $E$, Lesem MD, Jimerson DC. Resting metabolic rate of anorexia nervosa patients during weight gain. Am J Clin Nutr. 1994;60(5):666-75.

23. Golden NH, Katzman DK, Sawyer SM, Ornstein RM, Rome ES, Garber AK, et al. Update on the medical management of eating disorders in adolescents. J Adolesc Health. 2015;56(4):370-5.

24. Hay P, Chinn D, Forbes D, Madden S, Newton R, Sugenor L, et al. Royal Australian and New Zealand College of Psychiatrists clinical practice guidelines for the treatment of eating disorders. Aust N Z J Psychiatry. 2014; 48(11):977-1008.

25. Garber AK, Mauldin K, Michihata N, Buckelew SM, Shafer MA, Moscicki AB. Higher calorie diets increase rate of weight gain and shorten hospital stay in hospitalized adolescents with anorexia nervosa. J Adolesc Health. 2013; 53(5):579-84.

26. Golden NH, Keane-Miller C, Sainani KL, Kapphahn CJ. Higher caloric intake in hospitalized adolescents with anorexia nervosa is associated with reduced length of stay and no increased rate of refeeding syndrome. J Adolesc Health. 2013;53(5):573-8.

27. Redgrave GW, Coughlin JW, Schreyer CC, Martin LM, Leonpacher AK, Seide $M$, et al. Refeeding and weight restoration outcomes in anorexia nervosa: Challenging current guidelines. Int J Eat Disord. 2015;48(7):866-73.

28. Whitelaw M, Gilbertson H, Lam PY, Sawyer SM. Does aggressive refeeding in hospitalized adolescents with anorexia nervosa result in increased hypophosphatemia? J Adolesc Health. 2010;46(6):577-82.

29. Madden S, Miskovic-Wheatley J, Clarke S, Touyz S, Hay P, Kohn MR. Outcomes of a rapid refeeding protocol in Adolescent Anorexia Nervosa. J Eat Disord. 2015;3:8. 015-0047-1. eCollection 2015.

30. Leclerc A, Turrini T, Sherwood K, Katzman DK. Evaluation of a nutrition rehabilitation protocol in hospitalized adolescents with restrictive eating disorders. J Adolesc Health. 2013;53(5):585-9.
31. Willer MG, Thuras P, Crow SJ. Implications of the changing use of hospitalization to treat anorexia nervosa. Am J Psychiatry. 2005;162(12): 2374-6.

32. Madden S, Miskovic-Wheatley J, Wallis A, Kohn M, Lock J, Le Grange D, et al. A randomized controlled trial of in-patient treatment for anorexia nervosa in medically unstable adolescents. Psychol Med. 2015:45(2):415-27.

33. Le Grange D, Lock J, Agras WS, Moye A, Bryson SW, Jo B, et al. Moderators and mediators of remission in family-based treatment and adolescent focused therapy for anorexia nervosa. Behav Res Ther. 2012;50(2):85-92.

34. Le Grange D. Examining refeeding protocols for adolescents with anorexia nervosa (again): challenges to current practices. J Adolesc Health. 2013;53(5): 555-6.

35. Rigaud D, Brondel L, Poupard AT, Talonneau I, Brun JM. A randomized trial on the efficacy of a 2-month tube feeding regimen in anorexia nervosa: $A$ 1-year follow-up study. Clin Nutr. 2007:26(4):421-9.

36. Holliday MA, Segar WE. The maintenance need for water in parenteral fluid therapy. Pediatrics. 1957;19(5):823-32.

37. Parker EK, Faruquie SS, Anderson G, Gomes L, Kennedy A, Wearne CM, Kohn MR, Clarke SD. Higher Caloric Refeeding Is Safe in Hospitalised Adolescent Patients with Restrictive Eating Disorders. Journal of Nutrition and Metabolism. 2016;2016. https://www.ncbi.nlm.nih.gov/pmc/articles/ PMC4880718/.

38. Lock J, Le Grange D. Treatment manual for anorexia nervosa: A family-based approach. New York: Guilford Publications; 2015.

39. Fairburn CG. Cognitive behavior therapy and eating disorders. New York: Guilford Press; 2008.

40. Kuczmarski RJ, Ogden CL, Guo SS, Grummer-Strawn LM, Flegal KM, Mei Z, et al. 2000 CDC Growth Charts for the United States: methods and development. Vital Health Stat 11. 2002;246:1-190.

41. Institute of Medicine of the National Academies. Dietary reference intakes for energy, carbohydrate, fiber, fat, fatty acids, cholesterol, protein, and amino acids. Washington, DC: National Academic Press; 2005.

42. Raudenbush SW, Bryk AS. Hierarchical linear models: Applications and data analysis methods. Sage; 2002.

43. IBM Corp. IBM SPSS Statistics for Windows. Version 24 [software]. Armonk: IBM Corp.; 2016

44. Raudenbush SW, Bryk AS, Congdon R. HLM 7.01 for Windows. Version 7.01 [software]. Skokie: Scientific Software International, Inc.; 2013.

45. O'Connor G, Nicholls D. Refeeding hypophosphatemia in adolescents with anorexia nervosa: a systematic review. Nutr Clin Pract. 2013;28(3):358-64.

46. Le Grange D, Accurso EC, Lock J, Agras S, Bryson SW. Early weight gain predicts outcome in two treatments for adolescent anorexia nervosa. Int J Eat Disord. 2014;47(2):124-9.

47. Frisch MJ, Herzog DB, Franko DL. Residential treatment for eating disorders. Int J Eat Disord. 2006;39(5):434-42.

48. Toulany A, Wong M, Katzman DK, Akseer N, Steinegger C, Hancock-Howard $\mathrm{RL}$, et al. Cost analysis of inpatient treatment of anorexia nervosa in adolescents: hospital and caregiver perspectives. CMAJ Open. 2015;3(2):E192-7.

\section{Submit your next manuscript to BioMed Central and we will help you at every step:}

- We accept pre-submission inquiries

- Our selector tool helps you to find the most relevant journal

- We provide round the clock customer support

- Convenient online submission

- Thorough peer review

- Inclusion in PubMed and all major indexing services

- Maximum visibility for your research

Submit your manuscript at www.biomedcentral.com/submit 